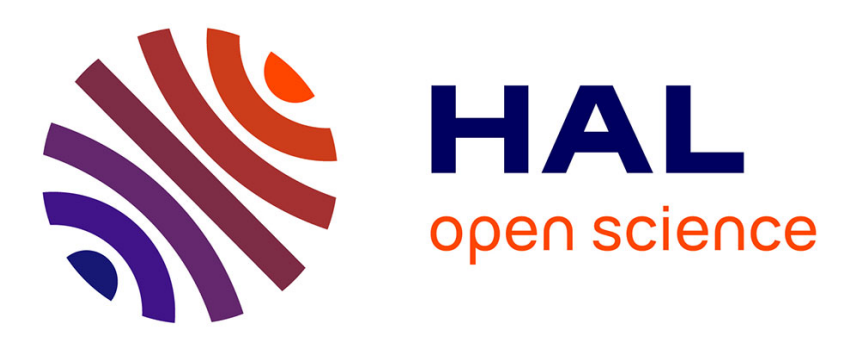

\title{
Caractérisation de MOS par des techniques d'analyse de surface en ultra-vide au cours de leur élaboration in situ
}

\author{
J.C. Dupuy, B. Vilotitch, A. Sibai
}

\section{To cite this version:}

J.C. Dupuy, B. Vilotitch, A. Sibai. Caractérisation de MOS par des techniques d'analyse de surface en ultra-vide au cours de leur élaboration in situ. Revue de Physique Appliquée, 1984, 19 (12), pp.965-970. 10.1051/rphysap:019840019012096500 . jpa-00245292

\section{HAL Id: jpa-00245292 https://hal.science/jpa-00245292}

Submitted on 1 Jan 1984

HAL is a multi-disciplinary open access archive for the deposit and dissemination of scientific research documents, whether they are published or not. The documents may come from teaching and research institutions in France or abroad, or from public or private research centers.
L'archive ouverte pluridisciplinaire HAL, est destinée au dépôt et à la diffusion de documents scientifiques de niveau recherche, publiés ou non, émanant des établissements d'enseignement et de recherche français ou étrangers, des laboratoires publics ou privés. 


\title{
REVUE DE PHYSIQUE APPLIQUÉE
}

\section{Caractérisation de MOS par des techniques d'analyse de surface en ultra-vide au cours de leur élaboration in situ (*)}

\author{
J. C. Dupuy, B. Vilotitch et A. Sibai \\ Institut National des Sciences Appliquées de Lyon, Laboratoire de Physique Industrielle, \\ 20, avenue A. Einstein, 69621 Villeurbanne Cedex, France
}

(Reçu le 16 mai 1984, accepté le 12 septembre 1984)

\begin{abstract}
Résumé. - Le silicium (100), nettoyé dans une enceinte ultravide, est caractérisé par spectroscopie Auger et diffraction d'électrons de très faible énergie. A partir d'un échantillon propre (diagramme de diffraction $\mathrm{Si}(100)$ $2 \times 1$, spectre Auger ne contenant que les transitions relatives au silicium en liaison 4-Si), l'oxydation est conduite in situ sous basse pression d'oxygène. L'examen des spectres Auger renseigne sur la nature et sur l'épaisseur des couches d'oxyde, dans la gamme de quelques $\AA$ à $30 \AA$. Une évaporation métallique permet de réaliser des structures MOS sans remise à l'air de l'échantillon. Le carbone, qui apparaît fréquemment comme principal contaminant de surface, n'est pas introduit par contamination à partir de l'atmosphère résiduelle lors de l'oxydation thermique. $\mathrm{Sa}$ localisation se situe au voisinage de l'interface $\mathrm{Si}_{-} \mathrm{SiO}_{2}$.
\end{abstract}

\begin{abstract}
Silicon (100) cleaned in an ultra-high vacuum chamber is characterized by Auger spectroscopy and Low Energy Electron Diffraction. Starting with a clean sample (diffraction pattern $2 \times 1$ for $\mathrm{Si}(100)$ and Auger spectra typical of 4-Si bonds), the oxidation is carried out in situ under low oxygen pressure. The thickness and nature of the oxide layer are studied by Auger spectroscopy in the 0-30 $\AA$ range and MOS device is realized by metal evaporation in situ without intermediate exposure of the sample to the air. Carbon which is the main surface contaminating agent is not introduced by the residual atmosphere during the oxidation process and is located near the $\mathrm{Si}_{-} \mathrm{SiO}_{2}$ interface.
\end{abstract}

\section{Introduction.}

L'oxydation du silicium constitue une étape particulièrement importante dans la réalisation des circuits intégrés. L'oxydation thermique, très utilisée, permet la réalisation de films isolants de bonne qualité et suffisamment homogènes. La croissance de l'oxyde thermique a donné lieu à de nombreuses études [1-4] qui concluent généralement à un régime de type linéaire-parabolique, la réaction d'oxydation se produisant à l'interface $\mathrm{Si}_{-} \mathrm{SiO}_{2}$ [5]. Dans la phase linéaire très rapide, la vitesse de croissance de l'oxyde est limitée par la réaction à l'interface; lors de la

(*) Communication présentée aux Journées du G.C.I.S., Toulouse les 15 et 16 décembre 1983. phase parabolique, c'est la diffusion de l'oxygène à travers la couche d'oxyde qui contrôle le phénomène d'oxydation. Le dopage du silicium n'affecte pas de façon significative la vitesse de croissance de l'oxyde, tant que sa valeur ne dépasse pas $10^{19}$ atomes $/ \mathrm{cm}^{3}$.

Cependant, la croissance de couches fines d'oxyde thermique d'épaisseur inférieure à $150 \AA$ se heurte encore à plusieurs problèmes :

- Présence de la couche d'oxyde natif, dont l'épaisseur peut atteindre jusqu'à $40 \AA$. Cet oxyde, de mauvaise qualité, doit être éliminé par traitement sous atmosphère contrôlée.

- Vitesse de croissance de l'oxyde trop élevée sous pression atmosphérique, ce qui rend difficile le contrôle du processus de réalisation de ces couches minces. 
- Présence d'une couche d'oxyde non stœchiométrique à l'interface $\mathrm{Si}_{-} \mathrm{SiO}_{2}$, qui est le siège de nombreux défauts. L'épaisseur de cet oxyde peut devenir comparable à celle de $\mathrm{SiO}_{2}$ pour les couches très minces de l'ordre de $30 \AA$ [6-9].

Les méthodes qui utilisent des techniques sous ultra-vide permettent de s'affranchir en partie de ces problèmes. Nous réalisons un nettoyage de la surface du silicium sous ultra-vide suivi d'une oxydation sous faible pression d'oxygène, sans remise à l'air intermédiaire. Dans ces conditions, la couche d'oxyde natif et les impuretés superficielles sont éliminées lors du nettoyage sous ultra-vide, l'échantillon une fois nettoyé sous $10^{-10}$ torr restant propre, vis-à-vis de la spectroscopie Auger, pendant plusieurs heures. Sous très faible pression d'oxygène, il est alors possible d'obtenir des couches d'oxyde d'épaisseur contrôlée dans la gamme des 20 à $50 \AA$, de façon parfaitement reproductible. Pour l'oxydation dans la gamme de pression d'oxygène de $10^{-4}$ à $10^{-2}$ torr, un modèle a été développé par Derrien et Commandré [10] qui conduit à un bon accord avec les cinétiques obtenues.

De nombreuses études, utilisant les spectroscopies ESCA ou Auger, ont porté sur l'adsorption de l'oxygène sur le silicium ou sur les premiers stades de l'oxydation; citons, à titre d'exemple, le déplacement et la modification du pic Si-2p en fonction du taux de recouvrement en oxygène $[11,12]$, et les modifications dans les transitions Auger KLL de l'oxygène [13].

Parmi les difficultés spécifiques qui apparaissent dans ce type d'expérimentation, il convient de signaler deux phénomènes :

- l'effet perturbateur du faisceau d'électrons primaires lors de l'analyse par spectroscopie Auger; une densité de courant trop élevée peut induire une oxydation du silicium sous des pressions d'oxygène de l'ordre de $10^{-6}$ torr $[14,15]$.

- La présence fréquente de carbone en tant qu'impureté de surface. Cette présence peut être imputée soit aux méthodes de nettoyage préliminaires à la mise sous vide de l'échantillon, soit au $\mathrm{CO}$ du vide résiduel qui peut être craqué par les divers filaments chauds dans l'installation (jauge, canon à électron). La présence de carbone en surface peut modifier les phénomènes d'oxydation à température ambiante sous faisceau électronique $[14,16]$, le rôle du $\mathrm{CO}$ résiduel semblant de peu d'importance pour l'oxydation thermique [17].

\section{Caractérisation des surfaces sous ultra-vide.}

2.1 TeChNiques UTILISÉES. - Nous effectuons toutes nos opérations d'analyse et de croissance des couches minces d'oxyde dans une enceinte ultra-vide, où la pression est inférieure à $10^{-9}$ torr lors des opérations de nettoyage et d'analyse. Un spectromètre Auger à miroir cylindrique (CMA) permet l'enregistrement des spectres Auger en mode dérivé, avec un bon rapport signal sur bruit dans la gamme d'énergie utilisée. Les transitions étudiées sont les transitions LVV du silicium, KLL de l'oxygène, et KLL du carbone qui apparaît comme contaminant principal de surface, avec l'oxygène. Un diffracteur d'électrons lents à optique de haute tension [18] nous a permis précédemment de vérifier l'état cristallographique de la surface du silicium nettoyé sous ultra-vide, reconstruit de façon typique en $\operatorname{Si}(100) 2 \times 1$ lorsque le taux d'impuretés en surface est suffisamment faible [19, 20].

2.2 Mode OPÉrATOIRE. - Le silicium (100) étudié est de type $n$ ou $\mathrm{p}$, dopé de $10^{15}$ à $10^{16}$ atomes $/ \mathrm{cm}^{3}$. Le type et le dopage n'ont aucune influence notable sur tous les résultats que nous avons obtenus [21]. Le nettoyage sous ultra-vide de la surface de l'échantillon peut être conduit de différentes manières; citons en particulier le bombardement ionique sous atmosphère d'oxygène, suivi par une évaporation de la couche d'oxyde ainsi formée [14]. Nous avons choisi la méthode classique de cycles de bombardements ioniques (argon, $500 \mathrm{eV}, 5 \mu \mathrm{A}$ ) et de recuits rapides vers $1000^{\circ} \mathrm{C}$. Cette méthode est généralement suffisante pour que l'échantillon soit parfaitement propre vis-à-vis de la spectroscopie Auger (pics KLL de l'oxygène et du carbone non discernables du bruit de fond) et bien structuré cristallographiquement (diagramme de diffraction d'électrons lents $2 \times 1$ ). Il faut toutefois noter que de simples recuits sont souvent suffisants pour nettoyer l'échantillon. L'oxygène s'élimine facilement, alors que la quantité de carbone initialement présent en surface varie beaucoup selon les échantillons; un nettoyage chimique [22] préalable apporte peu d'amélioration.

A partir d'une surface soit propre, soit comportant une quantité de carbone contrôlée, la croissance de la couche d'oxyde est conduite in situ. L'échantillon est porté à la température requise, l'oxygène est introduit dans l'enceinte sous des pressions de $10^{-4}$ à $10^{-1}$ torr en maintenant un léger flux pour éviter une contamination de l'atmosphère par désorption des parois [10]. Après refroidissement de l'échantillon, un pompage rapide est assuré par une cryopompe à l'hélium liquide, puis par une pompe ionique assistée d'un sublimateur de titane; le retour sous $10^{-9}$ torr se fait en quelques minutes. Après analyse, et avant toute autre opération, l'échantillon est de nouveau nettoyé sous ultra-vide par chauffage vers $1000^{\circ} \mathrm{C}$.

2. 3 INTERPRÉTATION DES SPECTRES AUGER. - Avant et après toute opération d'oxydation les spectres Auger sont effectués sur la même région de l'échantillon. Les transitions relatives au silicium sont bien connues [23], et se produisent aux énergies $92 \mathrm{eV}$ (silicium en liaison 4-Si), $82 \mathrm{eV}$ (en liaison $\mathrm{SiO}_{x}$ ), $78 \mathrm{eV}$ (en liaison $\mathrm{SiO}_{2}$ ), avec un décalage progressif vers les basses énergies pour $\mathrm{SiO}_{x}$ lorsque l'épaisseur d'oxyde augmente. Lorsque l'épaisseur d'oxyde dépasse 5 à $10 \AA$, le pic relatif à $\mathrm{SiO}_{x}$ disparaît. 
L'épaisseur des couches d'oxyde est évaluée à partir de l'affaiblissement du signal relatif à $\mathrm{Si}(92 \mathrm{eV})$ lors de la traversée de la couche d'oxyde. L'intensité du signal est alors de la forme :

$$
I=I_{0} \exp \left(-\frac{d}{\lambda}\right)
$$

$I_{0}$ étant le signal relatif à la surface propre, $d$ l'épaisseur de la couche d'oxyde, $\lambda$ le libre parcours moyen des électrons de $92 \mathrm{eV}$ dans l'oxyde. Nous avons choisi la valeur $\lambda=6,5 \AA$, ainsi que la technique de mesure des rapports pic-à-pic, proposées par Derrien [10]. Ceci permet la mesure d'épaisseur des couches d'oxyde jusqu'à $30 \AA$, de façon parfaitement reproductible.

\section{Résultats.}

3.1 CinétiQues D'oxydation. - Nous avons choisi d'illustrer ces cinétiques dans deux cas extrêmes $\left(5 \times 10^{-4}\right.$ torr d'oxygène, $550^{\circ} \mathrm{C}$, et $1 \times 10^{-1}$ torr d'oxygène $750^{\circ} \mathrm{C}$ ), la procédure étant celle décrite au paragraphe 2.2 .

Sous basse pression $\left(5 \times 10^{-4}\right.$ torr), les cinétiques ont été tracées à partir d'un échantillon propre vis-àvis de la spectroscopie Auger (Figs. 1 et 2), et à partir d'un échantillon contaminé par du carbone (Figs. 3 et 4). L'oxydation débute rapidement, puis devient extrêmement lente lorsque l'épaisseur de l'oxyde atteint $15 \AA$, cette quasi-saturation étant due à la faible pression d'oxygène et à la faible température, qui ne permettent pas une concentration d'oxygène en surface

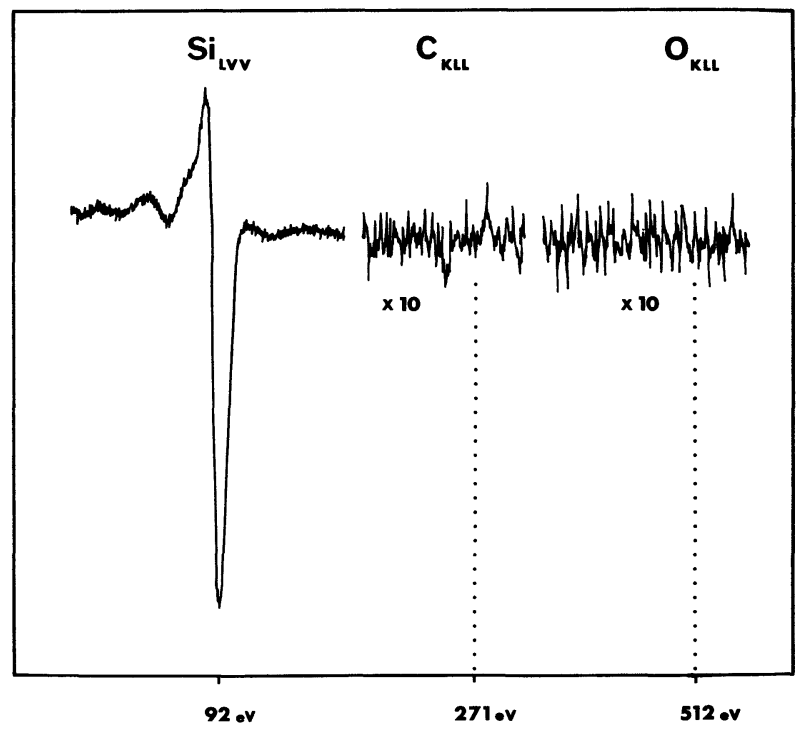

Fig. 1. - Spectre Auger enregistré en mode dérivé pour : $\mathrm{Si}_{\mathrm{LVV}}$ à $92 \mathrm{eV}, \mathrm{C}_{\mathrm{KLL}}$ à $271 \mathrm{eV}, \mathrm{O}_{\mathrm{KLL}}$ à $512 \mathrm{eV}$, avant oxydation. L'échantillon est propre vis-à-vis de la spectroscopie Auger : pic de $\mathrm{C}$ et $\mathrm{O}$ indiscernables.

[Derived Auger spectra before oxidation : $92 \mathrm{eV}$ for $\mathrm{Si}_{\mathrm{LVV}}$, $271 \mathrm{eV}$ for $\mathrm{C}_{\mathrm{KLL}}$ and $512 \mathrm{eV}$ for $\mathrm{O}_{\mathrm{KLL}}$. $\mathrm{C}$ and $\mathrm{O}$ peaks are undistinguishable.]

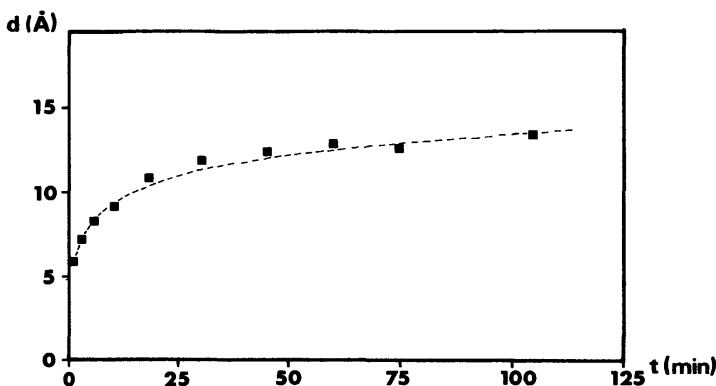

Fig. 2. - Cinétique d'oxydation d'un monocristal de Si(100) sous $5 \times 10^{-4}$ torr d'oxygène ultra pur à $550^{\circ} \mathrm{C}$. L'état initial de l'échantillon est décrit figure 1 . La courbe en pointillés représente le lissage par une fonction $\exp (\alpha d)-$ $\alpha d-1=\beta t$. $\alpha$ et $\beta$ étant fittés par les points expérimentaux (voir Réf. [10]).

[Kinetic oxidation of a $\mathrm{Si}(100)$ monocrystal under $5 \times 10^{-4}$ torr of oxygen at 550 ० $\mathrm{C}$. The initial state of the sample is shown on figure 1 . The dashed curve is a fitting by a function $\exp (\alpha d)-\alpha d-1=\beta t$ (see Ref. [10]).]

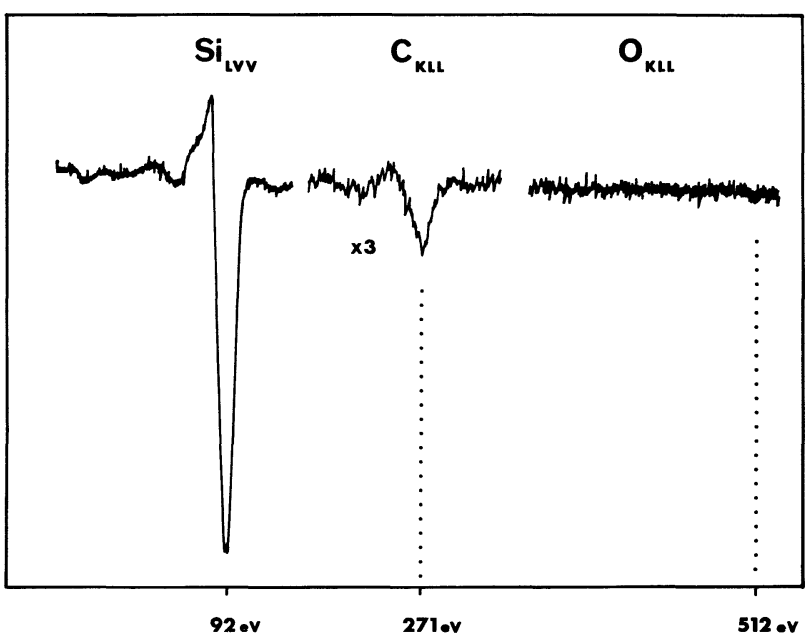

Fig. 3. - Spectre Auger enregistré en mode dérivé pour : $\mathrm{Si}_{\mathbf{L V V}}, \mathrm{C}_{\mathrm{KLL}}$ et $\mathrm{O}_{\mathbf{K L L}}$ avant oxydation d'un échantillon contaminé par des traces de carbone.

[Derived Auger spectra of a carbon contaminated sample (traces of $\mathrm{C}$ ) before oxidation : peaks $\mathrm{Si}_{\mathbf{L V V}}, \mathrm{C}_{\mathrm{KLL}}$ and $\left.\mathrm{O}_{\mathrm{KLL}} \cdot\right]$

suffisante ni un coefficient de diffusion assez élevé. Ce résultat est en bon accord avec ceux obtenus par Commandré et Derrien [10] sur le silicium (111).

La comparaison des courbes des figures 2 et 4 montre que le carbone initialement présent en surface ne semble pas jouer de rôle significatif sur la cinétique d'oxydation.

La figure 5 illustre la rapidité de la cinétique d'oxydation sous $10^{-1}$ torr à $750^{\circ} \mathrm{C}$. La température et la pression plus élevées que précédemment conduisent à obtenir une épaisseur d'oxyde de $30 \AA$ en $20 \mathrm{~min}$; audelà de cette épaisseur, il ne nous est plus possible 


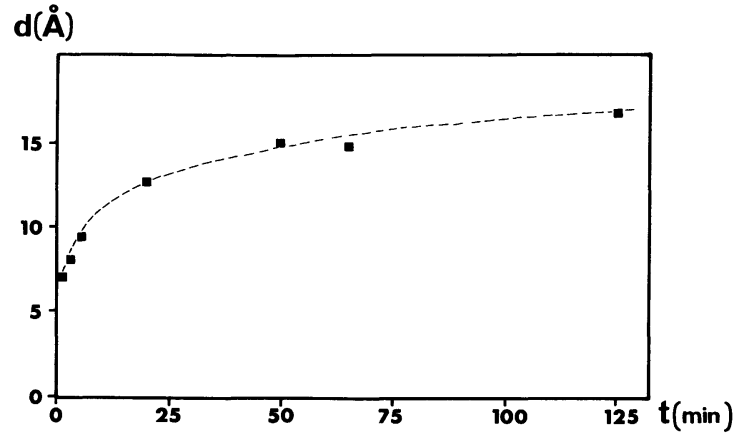

Fig. 4. - Cinétique d'oxydation d'un monocristal de Si(100) en présence de traces de carbone en surface $\left(5 \times 10^{-4}\right.$ torr, $\left.550^{\circ} \mathrm{C}\right)$. Etat initial de l'échantillon décrit figure 3.

[Kinetic oxidation of a $\mathrm{Si}(100)$ monocrystal contaminated by carbon surface traces under $5 \times 10^{-4}$ torr and $550^{\circ} \mathrm{C}$. The initial state of the sample is shown on figure 3.]

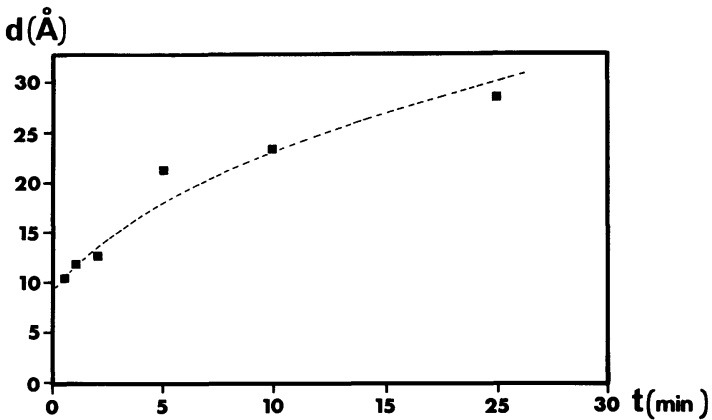

Fig. 5. - Cinétique d'oxydation d'un monocristal de $\mathrm{Si}(100)$ sous $10^{-1}$ torr à $750^{\circ} \mathrm{C}$ pour $d<30 \AA$.

[Kinetic oxidation of a $\mathrm{Si}(100)$ monocrystal under $10^{-1}$ torr of $\mathrm{O}_{2}$ and $750^{\circ} \mathrm{C}$ for $\left.d<30 \AA.\right]$

d'effectuer de mesure d'épaisseur in situ avec la technique utilisée, l'amplitude du signal Si LVV devenant trop faible pour être mesurée avec précision. L'ellipsométrie nous a montré après coup que des épaisseurs de l'ordre de $150 \AA$ pourraient être atteintes.

3.2 Localisation DU CARBONE. - Nous nous sommes efforcés de localiser le carbone, qui est souvent présent à la surface des échantillons même après nettoyage in situ [24]. Des travaux ont montré que le carbone reste concentré près de l'interface $\mathrm{Si}-\mathrm{SiO}_{2}$ après oxydation $[24,25]$, mais avec une extension de l'ordre de $100 \AA$ à $150 \AA$ des deux côtés de l'interface pour des oxydes épais.

Nous avons étudié la répartition du carbone dans les couches d'oxyde dont l'épaisseur ne dépasse pas $30 \AA$. L'oxydation a été conduite sous $5 \times 10^{-4}$ torr d'oxygène à $550^{\circ} \mathrm{C}$, pour des temps allant de $30 \mathrm{~s}$ à $2 \mathrm{~h}$, à partir d'échantillon contenant superficiellement du carbone en faible quantité (rapport des signaux
Auger pic-à-pic carbone/silicium $=6 / 100$ ). Afin d'éliminer toute influence d'un éventuel oxyde de carbone résiduel dans l'atmosphère, nous avons effectué au préalable les mêmes traitements thermiques sous une pression de $\mathrm{CO}$ de $5 \times 10^{-4}$ torr (Fig. 6) : aucune contamination supplémentaire par le carbone n'est apparue; de même à cette température de $550^{\circ} \mathrm{C}$, aucun enrichissement en surface dû à la diffusion d'un éventuel carbone présent en volume n'apparaît; le seul carbone que nous détectons est donc celui initialement présent à la surface de l'échantillon.

La figure 7 est relative à l'intensité du pic Auger du carbone à $271 \mathrm{eV}$ en fonction de l'épaisseur d'oxyde, au cours de l'oxydation; après chaque étape d'oxydation, le spectre Auger est enregistré, et l'épaisseur d'oxyde mesurée comme décrit au paragraphe 2.3. A partir du même échantillon oxydé, nous avons décapé progressivement la couche d'oxyde par bombardement ionique (ion argon, $600 \mathrm{eV}, 3 \mu \mathrm{A}$ ) et suivi l'amplitude du signal Auger du carbone. L'évolution du carbone en fonction de l'épaisseur d'oxyde est cohérente avec les résultats présentés figure 6 , malgré une dispersion et une incertitude plus grandes sur les points expérimentaux.

Dans les deux cas, on constate une diminution du signal Auger relatif au carbone en fonction de l'épaisseur d'oxyde dès que cette épaisseur est de l'ordre de 15 à $20 \AA$. Il n'y a donc pas de migration du carbone vers la surface libre de $\mathrm{SiO}_{2}$, mais il apparaît qu'une partie importante du carbone reste concentrée au voi-

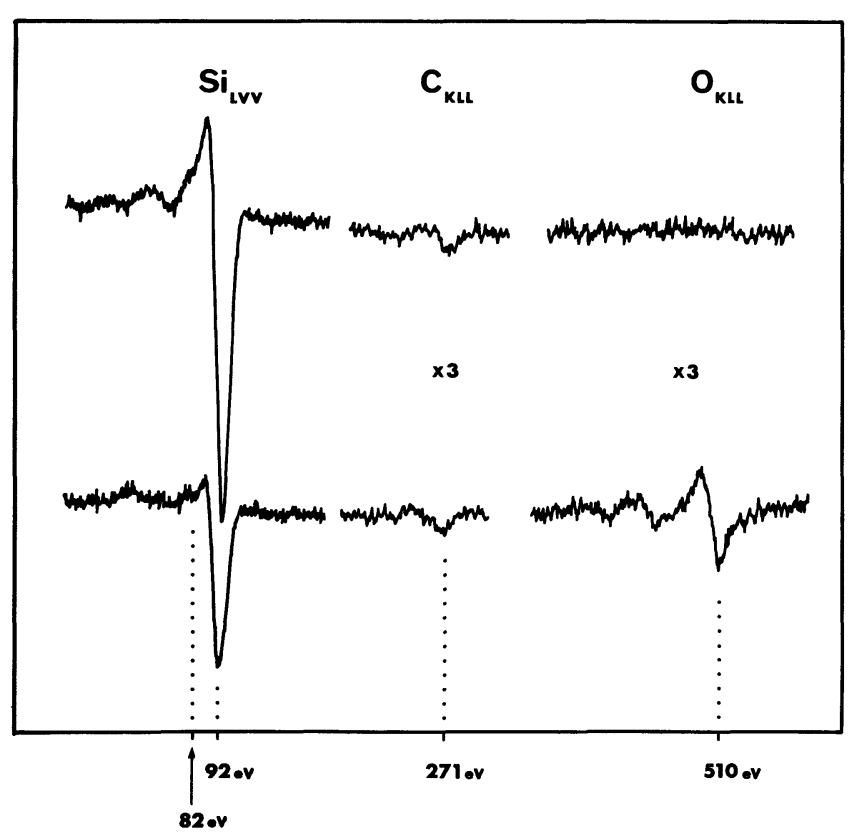

Fig. 6a. - Spectre Auger de $\mathrm{Si}_{\mathrm{LVv}}, \mathrm{C}_{\mathrm{KLL}}, \mathrm{O}_{\mathrm{KLL}}$ avant et après exposition sous $5 \times 10^{-4}$ torr de $\mathrm{CO}$ à $550^{\circ} \mathrm{C}$ pendant $20 \mathrm{~min}$.

[Derived Auger spectra, before and after exposure during $20 \mathrm{~min}$ to $5 \times 10^{-4}$ torr of $\mathrm{CO}$ at $550^{\circ} \mathrm{C}$.] 


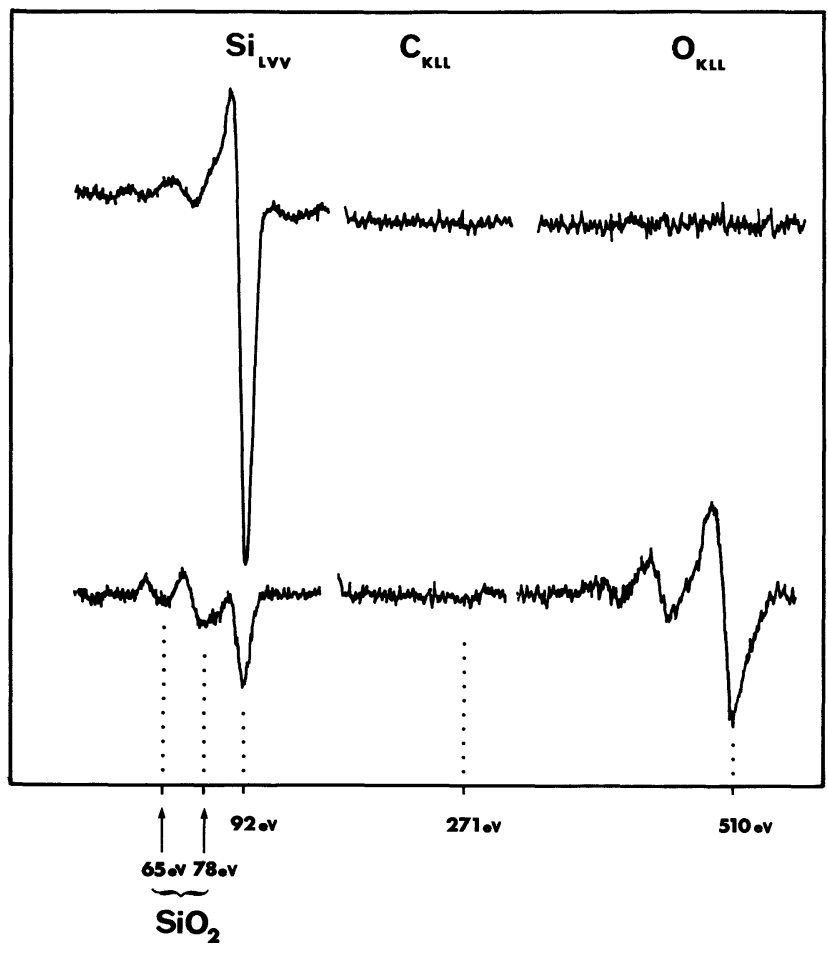

Fig. 6b. - Spectre Auger de $\mathrm{Si}_{\mathrm{LVV}}, \mathrm{C}_{\mathrm{KLL}}, \mathrm{O}_{\mathrm{KLL}}$ avant et après oxydation sous $5 \times 10^{-4}$ torr $\mathrm{O}_{2}$ à $550^{\circ} \mathrm{C}$ pendant $11 \mathrm{~min}$.

[Derived Auger spectra before and after exposure during $11 \mathrm{~min}$ to $5 \times 10^{-4}$ torr of $\mathrm{O}_{2}$ at $550^{\circ} \mathrm{C}$.]

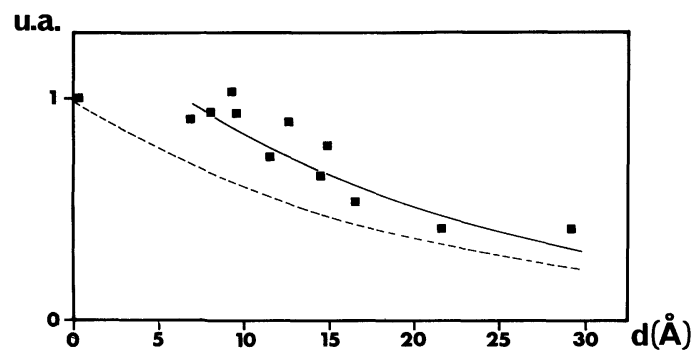

Fig. 7. - Evolution de l'intensité du signal Auger du carbone $(271 \mathrm{eV})$ en fonction de l'épaisseur d'oxyde. A titre de comparaison, nous avons tracé les courbes qui seraient obtenues avec une localisation exacte du carbone à la position $d=0$ (traits pointillés) et à la position $d=7 \AA$ (trait plein), à partir de $I=I_{0} \exp (-d / \lambda)$ avec l'hypothèse $\lambda=20 \AA$ pour les électrons de $271 \mathrm{eV}$.

[Variations of the carbon Auger signal intensity $(271 \mathrm{eV})$ versus the oxide thickness. For comparison we plotted the curves obtained with carbon located at $d=0$ (dashed line) and $d=7 \AA$ (full line), using the relation $I=I_{0} \exp (-d / \lambda)$ with the hypothesis $\lambda=20 \AA$ for the $271 \mathrm{eV}$ peak electrons.]

sinage immédiat de l'interface $\mathrm{Si}_{-} \mathrm{SiO}_{2}$ ainsi que le montre l'atténuation du signal Auger, qui peut être attribuée à son absorption lors de la traversée de la couche d'oxyde.
3.3 RÉAlisation DE MOS in situ. - Après oxydation par les méthodes décrites ci-dessus, une évaporation d'une grille métallique permet d'obtenir des structures MOS entièrement réalisées in situ. Pour des épaisseurs d'oxyde supérieures à $100 \AA$, l'aluminium nous donne de bonnes caractéristiques électriques. Cependant, pour des couches d'oxydes égales ou inférieures à $30 \AA$, la conductance des structures MOS est souvent trop élevée [26, 27]. Les phénomènes physiques parasites lors du dépôt métallique sont soit une réduction de la silice [28], soit une pénétration des atomes métalliques dans l'oxyde [29], suivant leur électronégativité. Le chrome ayant donné de bons résultats dans le cas de couches d'oxyde de $40 \AA$ [30], nous avons effectué des essais préliminaires, qui ont montré en spectroscopie Auger que ce métal se trouvait déposé à l'état oxydé (Fig. 8). Cependant, cette oxydation peut être due à l'atmosphère résiduelle, de l'ordre de $10^{-9}$ torr.

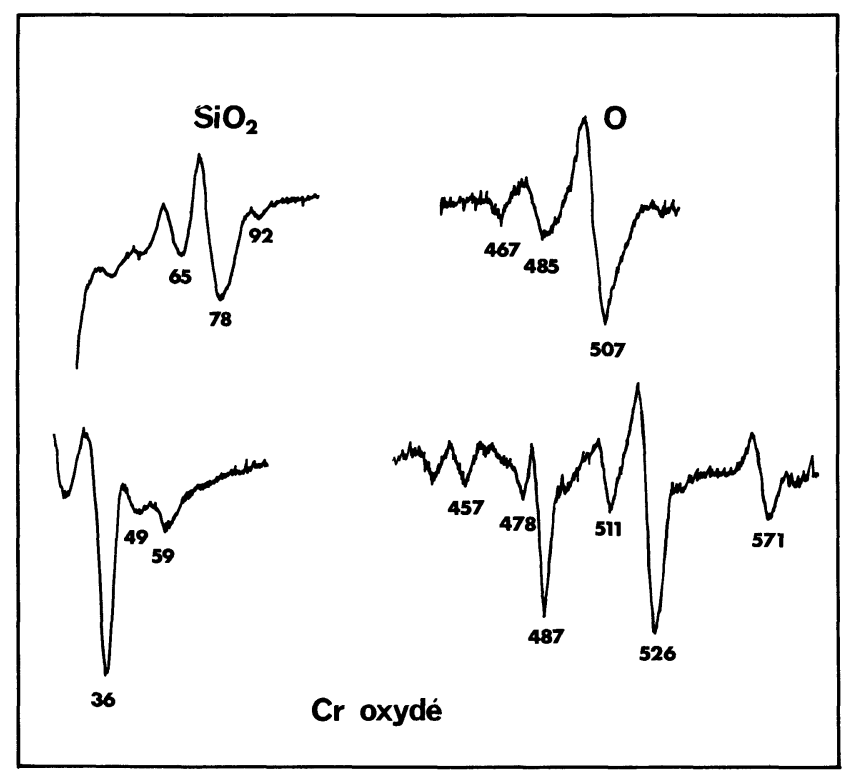

Fig. 8. - Spectre Auger enregistré en mode dérivé pour $\mathrm{Cr}, \mathrm{Si}, \mathrm{O}$ avant et après dépôt de chrome sur $\mathrm{SiO}_{2}$.

[Derived Auger spectra for $\mathrm{Cr}, \mathrm{Si}$ and $\mathrm{O}$ before and after chromium deposition on $\mathrm{SiO}_{2}$.]

\section{Conclusion.}

A partir d'un échantillon nettoyé sous ultra-vide et parfaitement propre vis-à-vis de la spectroscopie Auger, c'est-à-dire avec un taux d'impuretés en surface inférieur à 1/100 de monocouche, il est possible de réaliser in situ, de façon parfaitement contrôlée, des MOS dont l'épaisseur d'oxyde est inférieure ou égale à $30 \AA$. Le carbone, lorsqu'il est présent à la surface de l'échantillon, n'intervient pas sur les cinétiques d'oxydation, mais semble se localiser au voisinage de l'interface $\mathrm{Si}_{-} \mathrm{SiO}_{2}$. 
Pour des couches aussi minces obtenues à partir d'un échantillon ainsi nettoyé, l'interface $\mathrm{Si}_{-} \mathrm{SiO}_{2}$ est considéré comme généralement de l'ordre de quelques $\AA$ : le problème est alors posé au niveau de l'interface $\mathrm{SiO}_{2}$-métal, qui peut être élargi soit par réduction de $\mathrm{SiO}_{2}$, soit par pénétration du métal dans
$\mathrm{SiO}_{2}$. Nous nous attachons à poursuivre cette étude en la complétant par des caractérisations électriques systématiques.

\section{Remerciements.}

Cette étude est financée par le G.C.I.S.

\section{Bibliographie}

[1] Irene, E. A., J. Appl. Phys. 54, 9 (1983) 5416.

[2] Deal, B. E., Grove, A. S., J. Appl. Phys. 36, 12 (1965) 3770.

[3] Motr, N. F., in Insulating films on semiconductors (1979) Ed. G. G. Roberts, M. J. Morant (Inst. Phys. Conf. Ser. 50).

[4] Revesz, A. G., Evans, R. J., J. Phys. Chem. Solids 30 (1969) 551.

[5] Rigo S. et al., Journées G.C.I.S. Microélectronique Toulouse (Décembre 1983).

[6] Jackman, T. E., Mac Donald, J. R., Surf. Sci. 100 (1980) 35.

[7] Hattori, T., Nishino, T., Surf. Sci. 86 (1979) 555.

[8] Revesz, A. G., J. non Crystal. Solids 11 (1973) 309.

[9] Scwidtal, K., Surf. Sci. 77 (1978) 523.

[10] Derrien J., Commandre, M., Surf. Sci. 118 (1982) 32.

[11] Garner, C. M., et al., Phys. Rev. B 19, 8 (1979) 3944.

[12] Hollinger, G., Himpsel, F. J., J. Vac. Sci. Technol. A1 (1983) 640.

[13] Legare, P., et al., Surf. Sci. 68 (1977) 348.

[14] Tougaard, S., Morgen, P., Onsgaard, J., Surf. Sci. 111 (1981) 545.

[15] Carrière, B., Chouiyakh, A., Lang, B., Surf. Sci. 126 (1983) 495

[16] Dupuy, J. C., Journées G.C.I.S. Microélectronique, Toulouse (Juin 1982) (non publié).
[17] Joyce. B. A.. Neave. J. H.. Surf. Sci. 27 (1971) 499.

[18] Berger, C.. Dupuy. J. (.. Laydevani. L.. BerNARD, R., J. Appl. Phys. 48, 12 (1977), 5027.

[19] Dupuy, J. C., Laydevant L., J. Phys. E. : Sci. Instrum. 16 (1983) 217.

[20] Laydevant, L., Dupuy, J. C., Journées G.C.I.S. Microélectronique Toulouse (Juin 1981) (non publié).

[21] Dupuy, J. C., Sibai, A., Vilotitch, B. (à paraître).

[22] IshizaKa, A., NaKagawa, K., ShIRAKI, M.B.E.C.S.T. - 2 Tokyo (1982) 183.

[23] Lang, B., Scholler, P., Carrière, B., Surf. Sci. 99 (1980) 103.

[24] Wager, J. F., Wilmsen, C. W., J. Appl. Phys. 50, 2 (1979) 874.

[25] Haas, G. A., Gray, H. F., J. Appl. Phys. (1975) 46, 9 (1975) 3885.

[26] CommandRe, M., Thèse IDI 38123 Lyon (1981).

[27] Commandre, M., et al., In Insulating films on semiconductors 1981, ED. M. SCHUlz, G. PensL (Berlin : Springer Verlag) 1981.

[28] Roberts, S., Dobson, P. J., J. Phys. D. : Appl. Phys. 14 (1981) L 17.

[29] Bauer, R. S., Bachrach, R. Z., Brillson, L. J., Appl. Phys. Lett. 37, 11 (1980) 1006.

[30] KAR, S., Thèse Ph. D. 71-27, 720 Lehigh University, U.S.A. (1971). 